\title{
Nervio Interóseo Posterior: Origen, Localización y Trayecto Nervioso. Una Revisión
}

\author{
Posterior Interosseous Nerve: Origin, Location and Nerve Path: A Review
}

Pérez-Mérida, L. ${ }^{1,2}$; Aravena, R. ${ }^{3}$; Melo, R. ${ }^{4}$ \& Olave, E. ${ }^{5}$

\begin{abstract}
PÉREZ-MÉRIDA, L.; ARAVENA, R.; MELO, R. \& OLAVE, E. Nervio Interóseo posterior: origen, localización y trayecto nervioso. Una revisión. Int. J. Morphol., 39(6):1769-1775, 2021.

RESUMEN: El nervio interóseo posterior (NIP) ha sido utilizado como sinónimo ocontinuación inmediata del ramo profundo del nervio radial (RPNR) al emerger en el compartimiento posterior del antebrazo. Su origen tampoco es claro, describiéndose como nervio interóseo posterior a su trayecto proximal, intermedio o distal al músculo supinador. El objetivo de esta revisión es detallar la visión de diversos autores respecto al origen y trayecto del NIP, proponiendo una correcta terminología para estas estructuras. Se realizó una revisión bibliográfica de varios textos y de algunos artículos utilizados para la enseñanza de la anatomía humana, publicados entre los años 1800 y la actualidad. En la búsqueda, se determinaron criterios de inclusión que consideraban, anatomía humana, escritos en español, francés o inglés y que aludieran al NIP. Tras la exploración inicial se localizaron 18 libros, procedentes de Francia, Rusia, España, Argentina, Estados Unidos, Canadá, Reino Unido, Alemania, India y México. Una descripción del NIP más precisa, en cuanto al origen, trayecto y función, es aquella postulada por la vertiente francesa, correspondiendo a un origen terminal del ramo profundo del nervio radial, luego de emitir sus ramos musculares. Este delgado nervio transcurre adosado a la membrana interósea para luego avanzar por el cuarto compartimiento extensor, distribuyéndose en las articulaciones dorsales del carpo a quienes inerva sensitiva y propioceptivamente.
\end{abstract}

PALABRAS CLAVE: Nervio interóseo posterior; Síndrome del nervio interóseo posterior; Ramo profundo del nervio radial.

\section{INTRODUCCIÓN}

El Nervio Interóseo Posterior (NIP) ha sido asociado como una continuación directa del ramo profundo del nervio radial (RPNR) al emerger sobre el músculo supinador (Moore et al., 2018). Su origen no es muy claro, describiéndose como nervio interóseo posterior antes de atravesar el músculo supinador (Sunderland, 1946), atravesando el músculo supinador y siendo parte del síndrome del nervio interóseo posterior (Spinner, 1968; Regal Ramos, 2010), ó denominándolo así desde que emerge a partir del músculo en el compartimiento posterior de antebrazo (Drake et al., 2015).

Estas descripciones y localizaciones han ocasionado confusión en estudiantes, profesionales e investigadores que han abordado este importante nervio. Actualmente algunos textos de anatomía humana relacionan ambos térmi- nos como sinónimos (Ghosh, 2013), dejando en duda su denominación respecto al origen, trayecto y relaciones topográficas.

Desde el punto de vista clínico, se denomina nervio interóseo posterior a la división terminal del nervio radial, cuando este se divide en ramo superficial y profundo, habiendo inervado al músculo braquiorradial y extensor radial largo del carpo, luego el nervio atraviesa por las cabezas del músculo supinador, donde se forma la Arcada de Frohse (AF), siendo esta zona el lugar más frecuente del atrapamiento del nervio, constituyendo el "Síndrome del Interóseo Posterior", que se caracteriza por un déficit motor de la extensión de los dedos y del pulgar, asociado además a compromiso del abductor largo del pulgar con preservación de la sensibilidad brindada por el ramo superficial del nervio radial.

\footnotetext{
${ }^{1}$ Departamento de Anatomía y Medicina Legal, Facultad de Medicina, Universidad de Chile, Santiago, Chile.

${ }^{2}$ Departamento de Formación Transversal en Salud, Facultad de Ciencias de la Salud, Universidad Central de Chile, Santiago, Chile.

${ }^{3}$ Estudiante Medicina, Facultad de Medicina, Universidad de Chile, Santiago, Chile.

${ }^{4}$ Instituto de Neurocirugía, Departamento de Neurocirugía, Facultad de Medicina, Universidad de Chile, Santiago, Chile.

${ }^{5}$ Facultad de Medicina, Universidad de La Frontera, Temuco, Chile.
} 
En total existen cinco puntos de atrapamiento para el NIP a nivel del codo junto con su trayecto, que incluyen bandas fasciales fibrosas proximales a la cabeza radial, los vasos radiales recurrentes llamados "La correa de Henry" a la altura del cuello radial, el margen fibroso del extensor radial corto del carpo, la AF y el margen distal del supinador (Hohenberger et al., 2020).

Esta revisión detalla la visión de diversos autores respecto al origen y trayecto del NIP, dilucidando si su denominación se ajusta al descrito en su trayecto nervioso y proponiendo una correcta terminología para estas estructuras.

\section{MATERIAL Y MÉTODO}

Se realizó una revisión bibliográfica de varios textos y de algunos artículos utilizados para la enseñanza de la anatomía humana, publicados entre los años 1800 y la actualidad.

En primera instancia, se realizó una búsqueda de la bibliografía básica utilizada en la enseñanza de anatomía humana a través del "Portal de Libros de Bibliografía Básica", correspondiente a la biblioteca digital de la Universidad de Chile. Además, se utilizó la plataforma Google Scholar para encontrar otros libros utilizados que no se hallaron en la biblioteca virtual. Posteriormente, para expandir la pesquisa, se consultó el motor de búsqueda Pubmed, mediante las ecuaciones de búsqueda "Anatomy and old books". De esta manera, se analizó el artículo Old anatomy books (Louis, 1986) de donde se seleccionaron los libros de anatomía publicados en el siglo XIX.

En la búsqueda, se determinaron criterios de inclusión que consideraban, anatomía humana, escritos en español, francés o inglés y que aludieran al NIP. El principal criterio de exclusión fueron los atlas de anatomía sin texto y los libros de anatomía veterinaria.

Tras la exploración inicial se localizaron 18 libros: Traité d'anatomie humaine (Poirier \& Charpy, 1899), Anatomie des nerfs crâniens et rachidiens et du système grand sympathique chez l'homme (Hovelacque, 1927), Tratado de anatomía humana (Testut \& Latarjet, 1954), Anatomía Humana (Lockhart et al., 1965), Anatomía humana (Prives et al., 1984), Anatomía de Gardner (Gardner et al., 1989), Abrégé d'anatomie (Poirier et al., 1908), Anatomía humana descriptiva, topográfica y funcional (Rouvière et al., 2005), Anatomía humana (García-Porrero \& Hurlé, 2005), Prometheus, Texto y Atlas de Anatomía. Anatomía general y aparato locomotor (Schünke et al., 2005), Anatomía clíni- ca (Pró, 2012), Human anatomy for students (Ghosh), Anatomía humana (Quiroz, 2013), Gray. Anatomía para estudiantes (Drake et al.), Gray's Anatomy - The Anatomical Basis of Clinical Practice (Gray \& Vandyke, 2016), Anatomía con orientación clínica (Moore et al.), Anatomía humana (Latarjet \& Ruiz Liard, 2019) y Snell's clinical anatomy by regions (Wineski, 2019), procedentes de de Francia, Rusia, España, Argentina, Estados Unidos, Canadá, Reino Unido, Alemania, India y México.

De estos, se registraron antecedentes como: autoría, número de edición, editorial, año de publicación, ciudad de publicación, descripción sobre el NIP y número de las páginas en donde se encontraban información del NIP.

\section{RESULTADOS}

En primera instancia se han agrupado los textos franceses.

Para comenzar, Poirier \& Charpy mencionaron que "El nervio interóseo posterior se coloca entre el extensor largo de los dedos medialmente, el abductor largo de los dedos y el extensor corto de los dedos en el exterior; luego, en la unión del tercio medio y el tercio inferior del antebrazo, se vuelve más profundo y se aplica contra el ligamento interóseo. Cruveilhier le denomina nervio interóseo al tronco común del nervio que derivamos con este nombre y de las ramas que terminan en los músculos de la capa profunda; Por tanto, es importante señalar que el nervio interóseo sólo merece este nombre cuando se aplica contra la membrana interósea y que desciende fuera de los vasos interóseos".

Posteriormente, Poirier et al., en su texto mencionaron: “...Finalmente continúa en forma de una rama muy delgada, el nervio interóseo posterior, que viaja contra la membrana interósea y que puede seguirse hasta la cara dorsal de las articulaciones del carpo". Cabe destacar que en el apartado de "ramas terminales del nervio radial" describe al nervio interóseo posterior como el ramo terminal de la rama posterior del nervio radial.

Hovelacque señaló que "La rama de la capa profunda viaja primero entre las dos capas musculares que descansan hacia adelante sobre el abductor largo, se insinúa entre el abductor largo y a lo largo del pulgar que descansa hacia adelante sobre el extensor corto de los dedos, deja detrás de él, el extensor largo del pulgar y el extensor del dedo índice. A lo largo de este curso la rama se ubica fuera del plano axial del antebrazo, excepcionalmente puede ir acompañada de la arteria interósea posterior, pero casi siempre, ésta se desplaza en todo su recorrido entre los dos planos de los 
músculos de la cara posterior contigua en el flanco externo del músculo cubital posterior, por lo que abandona el nervio mientras se arrastra entre el abductor largo y el extensor largo. Debajo del extensor corto, el nervio toma el nombre de nervio interóseo y se aplica a la cara posterior del ligamento interóseo.

Testut \& Latarjet en su apartado de Ramas terminales del nervio radial describieron: "Rama posterior. La rama posterior o motora, la más importante de las dos ramas terminales del radial, se dirige hacia abajo, afuera y atrás, describiendo una especie de espiral alrededor del extremo superior del radio. En su trayecto cruza la cara anterior de la interlínea humero radial; luego, a 15 o 20 milímetros por debajo de esta interlínea, perfora la parte superior de la cara anterior del músculo supinador corto y penetra en su espesor. Desde entonces camina, en compañía de una arteria procedente de la recurrente radial anterior y de venas, entre los dos manojos del supinador corto. Corresponde así, sucesivamente, por medio de la capa profunda del supinador corto, sobre el que descansa, a la cara anterior del extremo superior del radio, luego a su cara externa y por último a su cara posterior. Desprendida del supinador corto, la rama profunda del radial llega entre los dos planos musculares de la región anterobraquial posterior y se expansiona en un ramillete de ramas que vienen a distribuirse por los músculos de la región posterior del antebrazo. En su trayecto entre los dos fascículos del supinador corto, la rama profunda del radial suministra dos o tres filetes a este músculo.

En su emergencia del supinador corto, algunas veces también en el espesor del músculo, la rama profunda del radial se divide en varios ramos: unos están destinados a los músculos de la capa superficial de la región antebraquial posterior y se designan con el nombre de ramos posteriores; otros se distribuyen por los músculos de la capa profunda y tienen el nombre de ramos anteriores; otro, en fin, largo y delgado, continúa el trayecto de la rama profunda hasta la muñeca con el nombre de nervio interóseo; se considera por algunos como el filete terminal de la rama motora. El nervio interóseo se aplica a la cara posterior del ligamento interóseo, se introduce en la cara posterior del carpo pasando por debajo del ligamento anular posterior, y se ramifica por la cara dorsal de las articulaciones de la muñeca y de los huesos del carpo".

Rouviére \& Delmas lo describieron como: "El ramo profundo del nervio radial atraviesa el músculo supinador para dirigirse a la región posterior del antebrazo. Alcanza el plano celular que separa las dos capas musculares e inerva todos los músculos que forman ambos planos. Después, muy adelgazado, recibe el nombre de nervio interóseo posterior y desciende hasta las articulaciones del carpo". Además, describe al RPNR, emergiendo del supinador cerca del margen inferior del músculo, por el intersticio que separa las dos capas que lo constituyen. Se sitúa luego entre los dos planos musculares de la región antebraquial posterior donde origina ramos posteriores y anteriores. El ramo profundo, considerablemente reducido después de haber proporcionado todos sus ramos colaterales, desciende entre los dos planos musculares, posteriormente al abductor largo y extensor corto del pulgar, para continuar luego posteriormente a la membrana interósea del antebrazo, anteriormente al extensor largo del pulgar y extensor del índice; penetra en la corredera osteofibrosa del extensor de los dedos y se ramifica en la cara dorsal de las articulaciones radiocarpiana y del carpo.

Latarjet \& Ruiz Liard señalaron que "Al emerger del supinador, el ramo profundo del nervio radial da los siguientes ramos... - Nervio interóseo antebraquial posterior: nervio propioceptivo que se aplica a la cara posterior de la membrana interósea, llega a la cara posterior del carpo, pasa profundo al retináculo extensor y se ramifica por la cara dorsal de las articulaciones carpianas y huesos del carpo".

En segunda instancia se agruparon los textos de origen estadounidense.

Gardner et al. señalaron que: "La rama profunda del nervio radial se origina por arriba o por abajo al epicóndilo, rodea el radio por fuera, pasando entre las porciones superficial y profunda del supinador corto, y continua como nervio interóseo posterior que inerva los músculos posteriores del antebrazo".

Wineski en Snell's Clinical Anatomy by Regions, mencionó que: "Rama profunda del nervio radial La rama profunda surge del nervio radial por delante del epicóndilo lateral del húmero en la fosa cubital (Fig. 3.29). Perfora el músculo supinador y se enrolla alrededor de la cara lateral del cuello del radio en la sustancia del músculo para alcanzar el compartimento posterior del antebrazo. Cuando el nervio emerge del músculo supinador en el compartimiento posterior, comúnmente se lo denomina nervio interóseo posterior. El nervio desciende en el intervalo entre los grupos de músculos superficiales y profundos y finalmente alcanza la superficie posterior de la articulación de la muñeca”.

Drake et al. mencionaron: "El ramo profundo del nervio radial, después de aparecer entre las dos cabezas del músculo supinador en el compartimento posterior del antebrazo, continúa como nervio interóseo posterior. ...Inerva el músculo supinador, y después sale del músculo como nervio interóseo posterior, situado entre los planos musculares superficial y profundo. El nervio interóseo posterior inerva 
el resto de los músculos del compartimento posterior y termina como ramos articulares, que pasan profundos al extensor largo del pulgar para llegar a la muñeca".

Gray \& Vandyke señalaron: "Nervio interóseo posterior". El nervio interóseo posterior es la rama terminal profunda del nervio radial (Figs. 46.9, 49.21, 49.29). Llega a la parte posterior del antebrazo pasando alrededor de la cara lateral del radio entre las dos cabezas del supinador (Figs. $49.25,49.26)$. Suministra al supinador tanto antes de entrar en el músculo como cuando lo atraviesa. A medida que emerge del supinador posteriormente, el nervio emite tres ramas cortas al extensor digitorum, extensor digiti minimi y extensor cubital del carpo, y dos ramas más largas: una rama medial al extensor largo del pulgar y extensor índice, y una rama lateral que inerva al abductor pulgar longus y extensor pollicis brevis. El nervio se encuentra al principio entre los músculos extensores superficiales y profundos, pero en el margen distal del extensor corto del pulgar pasa profundamente al extensor largo del pulgar y, disminuido a un hilo fino, desciende sobre la membrana interósea hasta el dorso del carpo. Los filamentos que surgen de su terminación aplanada irrigan los ligamentos y articulaciones del carpo. Las ramas articulares del nervio interóseo posterior inervan las articulaciones del carpo, radiocubital distal, y algunas intercarpianas e intermetacarpianas. Las ramas digitales irrigan las articulaciones metacarpofalángicas e interfalángicas proximales. La porción distal del nervio se encuentra en una vaina fascial separada en la cara radial, profunda, del cuarto compartimiento dorsal del retináculo extensor de la muñeca, donde se ubica profundo al extensor de los dedos y al extensor del índice".

Moore et al. describieron: "El nervio interóseo posterior es la continuación del ramo profundo del nervio radial, al que vemos aquí bifurcándose en dos ramos para inervar todos los músculos de vientre carnoso localizados completamente en el compartimento posterior del antebrazo". "Supinador...El ramo profundo del nervio radial pasa entre sus fibras y las separa en una parte superficial y una profunda, cuando se dirige desde la fosa del codo hasta la parte posterior del brazo. Cuando sale del músculo y se une a la arteria interósea posterior puede denominarse nervio interóseo posterior." Pag 455: "El ramo profundo penetra el músculo supinador y emerge en el compartimento posterior del antebrazo como nervio interóseo posterior. Se une a la arteria del mismo nombre para discurrir en un plano entre los extensores superficiales y profundos del antebrazo." Pag 464: "El ramo profundo del nervio radial, después de perforar el supinador, discurre por el plano fascial entre los músculos extensores superficiales y profundos, muy cerca de la arteria interósea posterior. Esta parte del nervio generalmente se conoce como el nervio interóseo posterior".
En tercer lugar, se analizaron textos que provienen de otras partes del mundo.

Lockhart et al. señalaron: "Del mismo modo del nervio radial y por delante del epicóndilo nace la rama terminal posterior o nervio interóseo (que va hacia afuera y atrás a través del supinador corto) y un filete inconstante para el segundo radial externo"

Prives et al. citaron: "Ramo profundo, que atraviesa el músculo supinador e inervándolo con un ramo, emerge en la parte posterior del antebrazo, donde inerva al músculo extensor radial breve del carpo y a todos los demás músculos posteriores del antebrazo. El nervio interóseo posterior, continuación del ramo profundo, desciende entre los extensores del pulgar hasta la articulación radiocarpiana, a la cual inerva. Por el trayecto que sigue el nervio radial se ve que éste inerva a todos los extensores del brazo y del antebrazo, y en este último, también a los músculos del grupo radial. En correspondencia con esto, inerva la piel en la parte extensora del brazo y del antebrazo. El nervio radial, continuación directa del tronco posterior, es como el nervio posterior del miembro superior".

Garcia-Porrero \& Hurlé describieron: "Nervio radial. La rama profunda (rama posterior), fundamentalmente motora, se desprende del radial a nivel de la interlínea articular, perfora el supinador, al que inerva, contornea por fuera el cuello del radio y pasa a la región antebraquial posterior, situándose entre el plano de los músculos extensores superficiales y el de los músculos profundos de esta región. A medida que desciende, emite ramos musculares para todos los músculos de la región: extensor cubital del carpo, extensor de los dedos y extensor del meñique en el plano superficial, y, en el plano profundo, los músculos, separador largo del pulgar, extensor corto del pulgar, extensor largo del pulgar y extensor del índice. El nervio se agota emitiendo el nervio interóseo posterior del antebrazo que desciende por detrás de la membrana interósea hasta el dorso del carpo (véase más adelante)".

Schunke et al. señalaron: “... el N. radial discurre entre el M. braquiorradial y el M. braquial distalmente por el codo, donde se divide en la R. profunda y la R. superficial. La R. profunda penetra en el M. supinador entre sus porciones superficial y profunda (canal supinador) y se dirige hacia la muñeca ya más fina como $\mathrm{N}$. interóseo (antebraquial) posterior".

Pró indicó: "Nervio radial. El ramo profundo sale del surco bicipital lateral y pasa entre las dos porciones del músculo supinador, para ingresar en el compartimento posterior del antebrazo donde emite ramos musculares para los 
músculos extensores del antebrazo (salvo el extensor radial largo del carpo) al igual que el abductor largo del pulgar. Luego da su ramo terminal, el nervio interóseo posterior, que se ubica entre la membrana interósea y los músculos extensores a nivel del tercio distal posterior del antebrazo llegando hasta la articulación radiocarpiana".

Quiroz señaló: “Además de los ramos anteriores y posteriores, del radial se desprende un ramo interóseo posterior que desciende hasta la cara posterior del carpo, pasando por delante del ligamento anular posterior. Se pierde en la cara dorsal de la articulación de la muñeca y en los huesos del carpo".

Ghosh describió: "Nervio radial. Rama terminal profunda (nervio interóseo posterior). Se enrolla alrededor de la cara lateral del radio y entre las fibras superficiales y profundas del supinador. Luego desciende en la parte posterior del antebrazo entre el grupo superficial y profundo de músculos extensores".

\section{DISCUSIÓN}

En general la mayoría de los libros actuales y "clásicos" revisados consideran al NIP como ramo terminal del RPNR, existiendo algunas diferencias con textos provenientes de Estados Unidos principalmente, los cuales los consideran como sinónimos o como directa continuación entre ambos nervios. Tal es el caso del libro "Anatomía con orientación clínica" (Moore et al.), el cual menciona derechamente como la continuación del RPNR y asociándolo a la Arteria Interósea Posterior otorgándole entonces el mismo nombre.

Por otro lado, en "Gray. Anatomía para estudiantes" (Drake et al.) se menciona que el NIP, inmediatamente luego de emerger entre las dos cabezas del músculo supinador en el compartimiento posterior de antebrazo. A continuación, el texto "Gray's Anatomy - The Anatomical Basis of Clinical Practice" (Gray \& Vandyke) menciona al NIP de la siguiente manera: "El nervio interóseo posterior es la rama terminal profunda del nervio radial. Llega a la parte posterior del antebrazo pasando alrededor de la cara lateral del radio entre las dos cabezas del supinador". En este caso, este texto menciona al NIP originado en la división del Nervio Radial a nivel del codo, siendo él mismo el que atraviesa el músculo supinador.

De igual manera "Snell's clinical anatomy by regions" (Wineski) menciona: "Cuando el nervio emerge del músculo supinador en el compartimiento posterior, comúnmente se lo denomina nervio interóseo posterior". En este caso volvemos a observar la misma situación anteriormente descrita, ya que se le otorga el nombre de NIP a la estructura luego de aparecer a nivel del músculo supinador. En el caso del texto "Human anatomy for students" (Ghosh) observamos un enunciado de subtítulo con la sinonimia habitual entre RPNR y NIP, este último encerrado en paréntesis de la siguiente manera: "Rama terminal profunda (nervio interóseo posterior)".

La totalidad de los libros franceses mencionan al NIP como estructura terminal del RPNR, luego de emitir ramos nerviosos a los diferentes músculos del compartimiento posterior de antebrazo. Así por ejemplo en "Traité d'anatomie humaine" (Poirier \& Charpy) mencionaron; "Por tanto, es importante señalar que el nervio interóseo sólo merece este nombre cuando se aplica contra la membrana interósea y que desciende fuera de los vasos interóseos."; contraponiendose a lo descrito por Moore et al. en alusión a la relación que se obtenía con la arteria interósea posterior.

Por su parte Rouvière et al. describieron que "Alcanza el plano celular que separa las dos capas musculares e inerva todos los músculos que forman ambos planos. Después, muy adelgazado, recibe el nombre de nervio interóseo posterior y desciende hasta las articulaciones del carpo.”; mientras que Latarjet \& Ruiz Liard, le otorga una función principalmente propioceptiva a este nervio en su distribución en la cara dorsal de los huesos del carpo.

Testut \& Latarjet, el gran libro de la anatomía humana mundial describe de la siguiente manera a este nervio: "En su emergencia del supinador corto, algunas veces también en el espesor del músculo, la rama profunda del radial se divide en varios ramos: unos están destinados a los músculos de la capa superficial de la región antebraquial posterior y se designan con el nombre de ramos posteriores; otros se distribuyen por los músculos de la capa profunda y tienen el nombre de ramos anteriores; otro, en fin, largo y delgado, continúa el trayecto de la rama profunda hasta la muñeca con el nombre de nervio interóseo; se considera por algunos como el filete terminal de la rama motora"

Todas estas descripciones son también observadas en libros de los siguientes autores, Pró, García-Porrero \& Hurlé y Quiroz.

Por otro lado, podemos concluir que este nervio no se asocia en todo su recorrido a la arteria interósea posterior (Moore et al.), sino que se separa de esta durante su recorrido, como exponen Hovelacque, Poirier \& Charpy.

Con respecto al ramo profundo del nervio radial, este se describe como un nervio motor en la mayoría de los tex- 
tos, pero no debemos olvidar que también corresponde a un nervio sensitivo ya que inerva las articulaciones del carpo (Gray \& Vandyke). Algo similar a lo encontrado en el estudio del nervio para el extensor radial corto del carpo en donde entre un $20 \%$ a $30 \%$ de la población se origina a partir del ramo superficial del nervio radial (Pérez-Mérida et al., 2018) no siendo exclusivamente sensitivo como ha sido descrito muchas veces.

La descripción de Hohenberger et al. se asemeja con nuestra postura, mencionándolo como un "ramo terminal del ramo profundo del nervio radial, el cual inerva el periostio del radio y ulna, la membrana interósea, así como la articulación radioulnar distal y las articulaciones de muñeca". Menciona además que en "la literatura internacional, el RPNR se denomina comúnmente como NIP”, lo que determina su utilización en las discusiones finales.

\section{CONCLUSIONES}

A la luz de lo expuesto por los diferentes textos, existen dos vertientes en cuanto a la descripción del origen y trayecto nervioso del NIP. Lo anterior podría explicar la continuidad como sinónimos que se le brindó al ramo profundo del nervio radial y nervio interóseo posterior durante mucho tiempo.

Creemos que una descripción del NIP más precisa, en cuanto al origen, trayecto y función, es aquella postulada por la vertiente francesa. Así entonces, la definición del NIP más adecuada corresponde a un origen terminal desde el ramo profundo del nervio radial, luego de emitir ramos musculares para el compartimiento posterior del antebrazo. Se profundiza, aplicándose sobre la membrana interósea, avanzando luego por el cuarto compartimiento extensor de muñeca, y finalizando su recorrido en la región dorsal de mano, específicamente inervando las articulaciones dorsales del carpo.

Esta inervación articular contribuye en la recepción de estímulos propioceptivos que colaboran en los movimientos de muñeca y mano. Desde el punto de vista clínico y quirúrgico de los nervios periféricos, se ha considerado el nervio interóseo posterior como un nervio eminentemente motor, no existiendo en la actualidad consideraciones respecto a este rol propioceptivo.

Por otro lado, es importante mencionar que este nervio no se asocia en todo su recorrido a la arteria interósea posterior, de la cual se separa para profundizarse bajo el músculo extensor corto del pulgar, y aplicándose sobre la membrana interósea. Así entonces, el ramo profundo del nervio radial tendría características motoras y sensitivas; algo muy parecido con lo expuesto en el ramo superficial del nervio radial, del cual en un porcentaje de la población, pueden desprenderse ramos motores específicamente para el músculo extensor radial corto del carpo, no siendo exclusivamente sensitivo.

Finalmente, y con todo lo descrito anteriormente, el nervio interóseo posterior posee un origen, trayecto y función diferente al ramo profundo del nervio radial, que es importante recalcar en el ámbito académico, neuroquirúrgico e investigativo.

PÉREZ-MÉRIDA, L.; ARAVENA, R.; MELO, R. \& OLAVE, E. Posterior interosseous nerve: Origin, location and nerve path. A review. Int. J. Morphol., 39(6):1769-1775, 2021.

SUMMARY: The posterior interosseous nerve (PIN) has been used as a synonym or immediate continuation of the deep branch of the radial nerve as it emerges in the posterior compartment of the forearm. Its origin is not clear either, being described as a posterior interosseous nerve to its proximal, intermediate or distal path to the supinator muscle. The objective of this review is to detail the vision of various authors regarding the origin and path of the PIN, proposing a correct terminology for these structures. A bibliographic review of several texts and some articles used for the teaching of human anatomy, published between the 1800s and the present day, was carried out. In the search, inclusion criteria were determined that considered human anatomy, written in Spanish, French or English and that alluded to the PIN. After the initial exploration, 18 books were located, coming from France, Russia, Spain, Argentina, the United States, Canada, the United Kingdom, Germany, India and Mexico. A more precise description of the PIN, in terms of origin, path and function, is that postulated by the French literature, corresponding to a terminal origin of the deep branch of the radial nerve, after emitting its muscular branches. This thin nerve runs attached to the interosseous membrane to then advance through the fourth extensor compartment, distributing itself in the dorsal carpal joints to which it innervates sensitively and proprioceptively.

KEY WORDS: Posterior Interosseous Nerve; Posterior Interosseous Nerve Syndrome; Deep Branch of the Radial Nerve.

\section{REFERENCIAS BIBLIOGRÁFICAS}

Drake, R.; Vogl, W. \& Mitchell, A. Gray. Anatomía para Estudiantes. 3a ed. Barcelona, Elsevier, 2015. pp.792.

García-Porrero, J. \& Hurlé, J. Anatomía Humana. Ciudad de México, McGraw Hill/Interamericana, 2005. pp.764.

Gardner, E.; Gray, D. \& O’Rahilly, R. Anatomía. 5ª ed. Ciudad de México, Interamericana. 1989. pp.134. 
Ghosh, B. Human Anatomy for Students. 2a ed. Nueva Delhi, Jaypee Brothers Medical Publishers, 2013. pp.31.

Gray, H. \& Vandyke, H. Gray's Anatomy - The Anatomical Basis of Clinical Practice. 41a ed. Philadelphia, Elsevier, 2016. pp.791, 860.

Hohenberger, G. M.; Schwarz, A. M.; Grechenig, P.; Maier, M. J.; Schwarz, U.; Kuchling, S.; Gänsslen, A. \& Weiglein, A. H. Morphology of the posterior interosseous nerve with regard to entrapment syndrome. Indian J. Orthop., 54(Suppl. 1):188-92, 2020.

Hovelacque, A. Anatomie des nerfs crâniens et rachidiens et du système grand sympathique chez l'homme. Arch. NeurPsych., 18(1):154-5, 1927.

Latarjet, M. \& Ruiz Liard, A. Anatomía Humana. 5a ed. Buenos Aires, Médica Panamericana, 2019. Vol. 1. pp.626.

Lockhart, R. L; Hamilton, G. F. \& Fyte, F. W. Anatomía Humana. Ciudad de México, Interamericana, 1965. pp.286.

Louis, R. Old anatomy books. Surg. Radiol. Anat., 8(3):153-4, 1986.

Moore, K.; Dalley, A. \& Agur, A. Anatomía con Orientación Clínica. 8a ed. Barcelona, Wolters Kluwer, 2018. pp.401, 451, 455, 464.

Pérez-Mérida, L.; Sousa-Rodriguez, C. F. \& Olave, E. Innervation of the extensor carpi radialis brevis muscle in Brazilian individuals: biometry of its origin and distribution. Int. J. Morphol., 36(3):799-805, 2018.

Poirier, P. \& Charpy, A. Traité d'Anatomie Humaine. Paris, Masson, 1899. Vol. 3. pp.1044.

Poirier, P.; Charpy, A. \& Cunéo, B. Abrégé d'Anatomie. Paris, Masson, 1908. Vol. 2. pp.1004.

Prives, M.; Lisenkov, N. \& Bushkovich, V. Anatomía Humana. 5a ed. Moscú, Mir, 1984. Vol. 3. pp.270.

Pró, E. Anatomía Clínica. Buenos Aires, Médica Panamericana, 2012. pp.795.

Quiroz, F. Anatomía Humana. 43ª ed. Ciudad de México, Porrúa, 2013. pp.455.

Regal Ramos, R. J. Neuropatía compresiva del nervio interoseo posterior a nivel del codo (síndrome de la arcada de frohse) ¿debe incluirse en el listado de enfermedades profesionales?. Med. Segur. Trab., 56(220):24856,2010

Rouvière, H.; Delmas, V. \& Delmas, A. Anatomía Humana. 11a ed. Barcelona, Masson, 2005. Vol. 3. pp.219, 220, 261.

Schünke, M.; Schulte, E.; Schumacher, U.; Voll, M. \& Wesker, K. Prometheus, Texto y Atlas de Anatomía. Anatomía General y Aparato Locomotor. Buenos Aires, Médica Panamericana, 2005. Vol. 1. pp.323.

Spinner, M. The arcade of Frohse and its relationship to posterior interosseous nerve paralysis. J. Bone Joint Surg. Br., 50(4):809-12, 1968.

Sunderland, S. Metrical and non-metrical features of the muscular branches of the radial nerve. J. Comp. Neurol., 85:93-111, 1946.

Testut, L. \& Latarjet, A. Tratado de Anatomía Humana. $9^{\mathrm{a}}$ ed. Barcelona, Salvat,1954. Vol. 3. pp.292-3.

Wineski, L. Snell's Clinical Anatomy by Regions. 10a ed. Philadelphia, Wolters Kluwer, 2019. pp.351.

\author{
Dirección para correspondencia: \\ Luis Pérez-Mérida, Mg.Cs. Morfología \\ Departamento de Anatomía y Medicina Legal \\ Universidad de Chile \\ Santiago \\ CHILE
}

Email: luis.perez.m@uchile.cl

Recibido : 26-07-2021

Aceptado: 28-10-2021 